\title{
Transfer Learning based Impedance Identification of Voltage Source Converters
}

\author{
Mengfan Zhang, Student Member, IEEE, Xiongfei Wang, Senior Member, IEEE, Qianwen Xu, Member, IEEE
}

\begin{abstract}
The black-box impedance of the voltage source converters (VSCs) can be directly identified at the converter terminal without access to its internal control details, which greatly facilitates the converter-grid interactions. However, since the limited impedance data amount in practical industrial applications, the existing impedance identification methods cannot accurately capture characteristics of the impedance model at various operating scenarios, which is the indicators of the VSCs system stability at the changing profiles of renewables and loads. In this paper, a transfer learning based impedance identification is proposed to fill this research gap. This method can significantly reduce the required data amount used in impedance identification so that the black-box impedance-based stability method could be applied for the practical industrial application. The comparison results confirm the accuracy of the impedance model obtained by this transfer learning based impedance identification method.
\end{abstract}

\section{INTRODUCTION}

Voltage source converters (VSCs) are increasingly used as the interface of the grid and renewables, which makes modern power grid more flexible, sustainable and efficient [1]. However, the interaction of the VSCs and grid impose new challenges to system stability [2], [3].

The impedance-based method is widely applied for stability analysis of VSC systems since it does not need to formulate models of the whole system. Instead, it predicts the system stability based on the impedance ratio of the equivalent converter impedance and the equivalent grid impedance [4], [5]. Considering the asymmetry caused by the phase locked loop (PLL) of the VSC, the multi-input multi-output (MIMO) impedance model is used to evaluate the grid-connected power system stability in the frequency domain using the generalized Nyquist criterion (GNC). A typical application is reported in [6], a $d q$-frame impedance model of the grid-connected VSC is derived, and the grid-converter interaction is then characterized with the equivalent grid impedance and the derived VSC impedance.

However, in practical industry applications, since no or limited information of the internal control system is available from the vendors, the impedance model of inverter systems cannot be obtained with the theoretical derivation. The impedance measurement is thus increasingly applied in practical stability analysis without intellectual property (IP) contamination. An impedance- based stability analysis is reported in [7], the measured grid-connected VSC impedance and the known grid impedance are used for the stability estimation of the grid-converter system. Yet, the existing impedance-based stability analysis methods are conducted only at the stiff operating scenario. Since the VSC is inherently a nonlinear system when the phase-locked loop (PLL) and the outer control loop are considered, the measured impedance of converters will change along with the operating point [8]. In order to predict the stability of the interaction system in different operating points, and considering the fluctuations of renewables and loads in the system, there is always a demand for the impedance of VSC in varying operating points.

To address this challenge, few methods are reported in previous literature. A polytopic model for dc-dc converters is reported in [9], where the identified small-signal models at multiple operating points are combined with weighting functions, and thus a multi-operating-point (MOP) impedance model can be obtained. Yet this model can just predict the impedance at limited operating points and hard to guarantee the accuracy. An improved method is to adapt the artificial neural network (ANN) technique to generate the MOPimpedance model with the measured impedance [10]. The ANN based impedance model trained with the measured impedance can describe the characteristics of the VSC at varying operating points. Nevertheless, the impedance data used in the model training need to be measured by repeated experiments with different operating points, as the operating point is the four-dimension vector, it is unrealistic to measure enough data for model training in field applications. The limited data amount will consequently introduce the overfitting phenomena that are harmful to the accuracy of the trained impedance model [11] and then affect the accuracy of stability estimation.

To overcome the bottleneck imposed by limited data amount in practical impedance identification, this paper proposes a transfer learning based impedance identification method. Transfer learning is an effective technique to deal with the data limitation issue in computer science by transferring the knowledge learned while solving one problem to solving another related problem [12]. Inspired by this concept, we propose a new physics based transfer learning method that if a neural network structure can be constructed in a way to resemble the inherent structure of the physical model of VSC, the accuracy of the trained models can be improved with limited data amount. The proposed method consists of following steps: First, the analytical model of the VSC is derived and an ANN structure is constructed based on the achieved analytical model. Next, numerical offline tests are conducted to generate the large amount impedance data, which are fed into the constructed ANN structure to generate the 


\section{IEEE TechRxiv}

offline impedance model. Then, based on transfer learning technique, the offline impedance model is transferred to different but related VSCs by iteratively minimize the marginal and conditional distribution differences between the trained data and unknown data. Thus, the online impedance model is generated with the limited field measured data.

The remainder of the paper is organized as follows: The analytical model of impedance is extracted based on the general control scheme of VSC with PLL and current control loops included. With the structure model and the training data, the proposed transfer learning-based impedance identification method is developed to obtain the VSC impedance model covering a wide range of operating points. Finally, comparative case studies are provided that confirm the effectiveness of the proposed method.

\section{GRID-CONNECTED VSC SYSTEM MODELING}

In real industrial projects, the circuit and control structures of VSCs are usually known while the detail control parameters cannot be accessed. Fig. 1 shows the typical diagram of threephase grid-connected VSC with the PLL and current control loop, where $L$ and $R_{L}$ denote the filtered inductor and its parasitic resistor respectively, $v_{a b c}$ and $i_{a b c}$ are the sampled three-phase voltages and currents at point of common coupling (PCC), $V_{o}$ and $V_{p c c}$ represent the output voltage and PCC voltage, $\theta$ denotes the synchronization phase generated by the PLL, I represent the inverter output current. The current control is realized with proportional integral (PI) controller in the $d q$-frame.

Fig. 2 shows the detailed control diagram of the three-phase VSC with current control and synchronous reference frame (SRF) PLL. The filter plant can be represented by the following equation:

$$
Y_{p}(s)=Y_{o}(s)=\frac{1}{L \cdot s+R_{L}}
$$

$G_{i}(s)$ is transfer function of the PI current controller, where $k_{p}$ and $k_{i}$ denote the proportional gain and integral gain, which is written as:

$$
G_{i}(s)=k_{p}+\frac{k_{i}}{s}
$$

$G_{d e l}(s)$ represents the time delay of the digital control system [13], which consists of the computational delay $\left(T_{s}\right)$

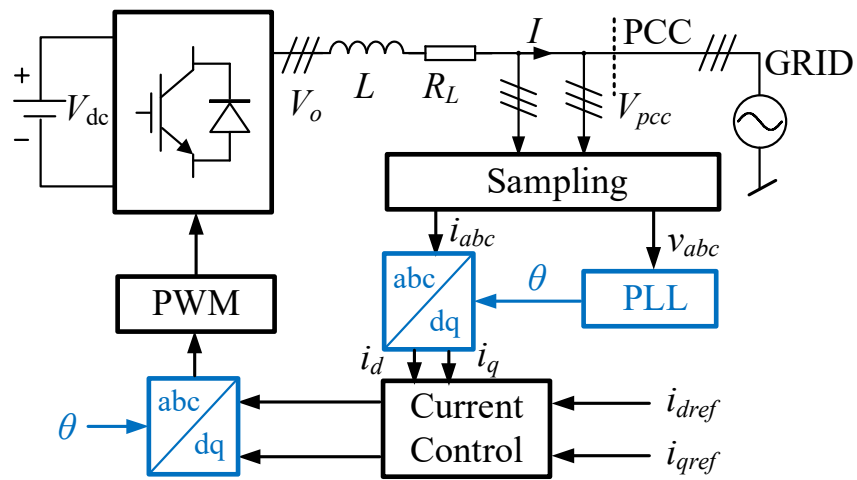

Fig. 1. Three-phase grid-connected VSC system with current control and PLL.

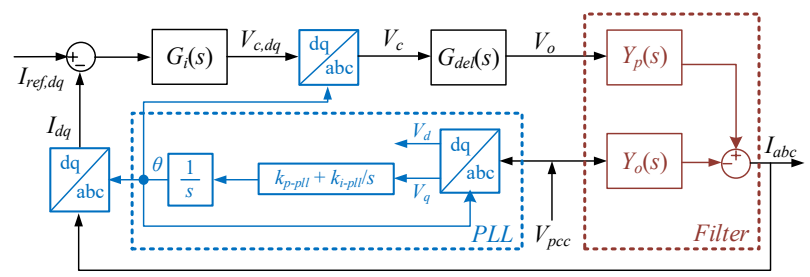

Fig. 2. Control diagram of grid-connected VSC with current controller and PLL.

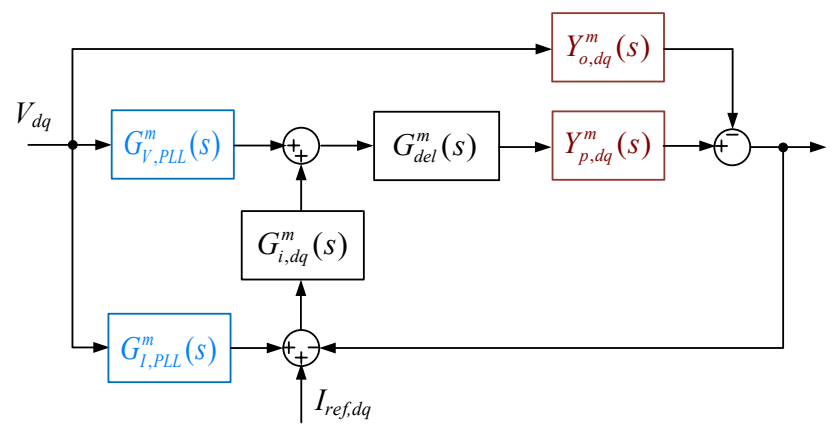

Fig. 3. Block diagram of small-signal transfer matrices of the grid-connected VSC system.

and pulse width modulator (PWM) delay $\left(0.5 T_{s}\right)$, where $T_{s}$ is the sampling period. The time delay is shown below:

$$
G_{d e l}(s)=e^{-1.5 T_{s} \cdot s}
$$

The SRF-PLL block can be linearized as a second-order dynamic system, which is given as [14]

$$
\begin{gathered}
\Delta \theta=G_{P L L}(s) \Delta V_{q} \\
G_{P L L}(s)=\frac{s \cdot k_{p-p l l}+k_{i-p l l}}{s^{2}+\left(s \cdot k_{p-p l l}+k_{i-p l l}\right) V_{d}}
\end{gathered}
$$

where $k_{p-p l l}$ and $k_{i-p l l}$ are the proportional gain and integral gain in PLL, respectively. The $d q$-transformations used in the current control are linearized as follows:

$$
\begin{aligned}
I_{d q} & =I \cos \theta+i \cdot I \sin \theta \\
& =(I+\Delta I) \cos (\Delta \theta+\theta)+i \cdot(I+\Delta I) \sin (\Delta \theta+\theta) \\
\rightarrow & \Delta I_{P L L, d q}=i \cdot \Delta \theta \cdot I_{s, d q} \\
V_{d q} & =V \cos \theta+i \cdot V \sin \theta \\
& =(V+\Delta V) \cos (\Delta \theta+\theta)-i \cdot(V+\Delta V) \sin (\Delta \theta+\theta) \\
\rightarrow & \Delta V_{P L L, d q}=i \cdot \Delta \theta \cdot V_{s, d q}
\end{aligned}
$$

where $I_{s, d q}$ and $V_{s, d q}$ denote the steady-state complex space vectors of current and voltage respectively, $\Delta I_{P L L, d q}$ and $\triangle V_{P L L, d q}$ denote the dynamic effects of the PLL on the current and the voltage of the VSC, respectively. Substituting (4) into (6) and (7) leads to

$$
\begin{aligned}
& \Delta I_{P L L, d q}=i \cdot G_{P L L}(s) \Delta V_{q} \cdot I_{s, d q} \\
& \Delta V_{P L L, d q}=i \cdot G_{P L L}(s) \Delta V_{q} \cdot V_{s, d q}
\end{aligned}
$$

The transfer functions with the additional superscript "m" represent the symmetric transfer matrices, which are shown below: 


$$
\begin{gathered}
Y_{p, d q}^{m}(s)=Y_{o, d q}^{m}(s)=\frac{1}{L\left(s^{2}+\omega_{1}^{2}\right)}\left[\begin{array}{cc}
s & \omega_{1} \\
\omega_{1} & s
\end{array}\right] \\
G_{i, d q}^{m}(s)=\left[\begin{array}{cc}
G_{i}(s) & 0 \\
0 & G_{i}(s)
\end{array}\right] \\
G_{d e l}^{m}(s)=\left[\begin{array}{cc}
G_{d e l}(s) & 0 \\
0 & G_{d e l}(s)
\end{array}\right]
\end{gathered}
$$

where $\omega_{1}$ is the grid fundamental frequency. The PLL effects are modeled by two transfer matrices

$$
\begin{aligned}
& {\left[\begin{array}{l}
\Delta I_{P L L, d} \\
\Delta I_{P L L, q}
\end{array}\right]=\left[\begin{array}{cc}
0 & -G_{P L L}(s) I_{s, q} \\
0 & G_{P L L}(s) I_{s, d}
\end{array}\right]\left[\begin{array}{l}
\Delta V_{d} \\
\Delta V_{q}
\end{array}\right]=G_{I, P L L}^{m}\left[\begin{array}{l}
\Delta V_{d} \\
\Delta V_{q}
\end{array}\right]} \\
& {\left[\begin{array}{l}
\Delta V_{P L L, d} \\
\Delta V_{P L L, q}
\end{array}\right]=\left[\begin{array}{cc}
0 & -G_{P L L}(s) V_{s, q} \\
0 & G_{P L L}(s) V_{s, d}
\end{array}\right]\left[\begin{array}{l}
\Delta V_{d} \\
\Delta V_{q}
\end{array}\right]=G_{V, P L L}^{m}\left[\begin{array}{l}
\Delta V_{d} \\
\Delta V_{q}
\end{array}\right]}
\end{aligned}
$$

where $I_{s, d}, I_{s, q}$ and $V_{s . d}, V_{s, q}$ denote the steady-state complex space vectors of current and voltage in $d$-axis and $q$-axis, respectively. Thus the block diagram of small-signal transfer matrices of the grid-connected VSC system is derived as shown in Fig. 3.

The closed-loop current response is derived as:

$$
\begin{aligned}
& {\left[\begin{array}{l}
I_{d} \\
I_{q}
\end{array}\right]=G_{c l, d q}^{m}(s)\left[\begin{array}{c}
I_{r e f, d} \\
I_{r e f, q}
\end{array}\right]} \\
& -\left\{\left[I^{m}+T_{d q}^{m}(s)\right]^{-1} Y_{t o, d q}^{m}(s)-G_{c l, d q}^{m}(s) Y_{P L L}^{m}(s)\right\}\left[\begin{array}{c}
V_{d} \\
V_{q}
\end{array}\right]
\end{aligned}
$$

where $I_{m}$ is the unitary diagonal matrix, the other transfer functions are shown below:

$$
\begin{aligned}
& Y_{t o, d q}^{m}(s)=Y_{p, d q}^{m}(s)-Y_{p, d q}^{m}(s) G_{d e l}^{m}(s) G_{V, P L L}^{m}(s) \\
& T_{d q}^{m}(s)=Y_{p, d q}^{m}(s) G_{d e l}^{m}(s) G_{c, d q}^{m}(s) \\
& G_{c l, d q}^{m}(s)=\left[I^{m}+T_{d q}^{m}(s)\right]^{-1} T_{d q}^{m}(s)
\end{aligned}
$$

Thus, the closed-loop admittance matrix of VSC can be derived as:

$$
Y_{t c l, d q}^{m}(s)=\left[I^{m}+T_{d q}^{m}(s)\right]^{-1} Y_{t o, d q}^{m}(s)-G_{c l, d q}^{m}(s) Y_{P L L}^{m}(s)
$$

As shown in (15), if the control structure of the VSC is fixed, the converter admittance model is dependent by the steady state operating point, frequency and the control parameters. In piratical industry applications, the detailed control parameters are not available in real application, so that the admittance shown in (15) is a structural model with the unknown control parameters.

\section{TRANSFER LEARNING BASED IMPEDANCE IDENTIFICATION}

Based on the admittance model derived in Section II, a transfer learning based impedance identification method is proposed, consisting of knowledge based offline phase and real-time measurement based online phase.

A. Statement

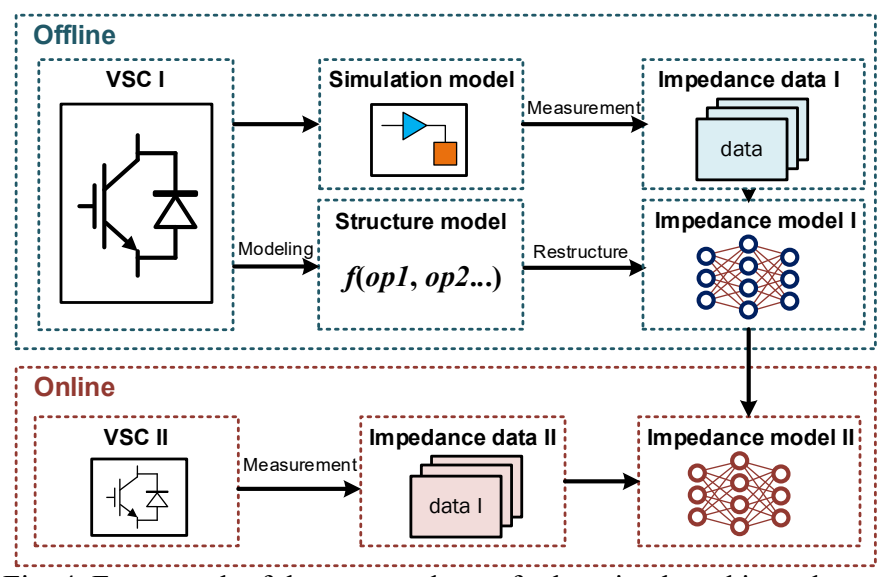

Fig. 4. Framework of the proposed transfer learning based impedance identification method.

\section{1) Dataset structure statement}

In industrial applications, the steady state operating points i.e., $I_{s, d q}$ and $V_{s, d q}$ are variable in different operating scenarios, the closed-loop admittance thus are variable. Due to the admittance model derived in (15), the admittance model is affected by the operating point. Thus the structure of multioperating-point admittance model is established, where the operating point of the VSC in $d q$-frame, i.e., $\left(V_{d}, V_{q}, I_{d}, I_{q}\right)$ and frequency $f_{p}$ are selected as the input of the model to reveal the operating-point-dependent and frequency-dependent feature. The magnitude and the phase of admittance in the $d q$-frame are selected as the outputs of the model.

\section{2) Problem statement}

As mentioned in section II, for the VSCs provided by different vendors, the control structure is known while the control parameters are uncertain. The purpose of proposed method is to utilize the impedance model trained by measured data and the analytical model for the VSCs with the different control parameters that are not learned by the model before.

The domain data consists of a feature space of input $X$ and a marginal probability distribution $P(\boldsymbol{X})$. Denoted by $D_{s}=\left\{\left(\boldsymbol{x}_{s 1}\right.\right.$, $\left.\left.\boldsymbol{y}_{s 1}\right), \ldots,\left(\boldsymbol{x}_{s n}, \boldsymbol{y}_{s n}\right)\right\}$ the simulated source-domain labeled data, where the input $\boldsymbol{X}_{s}$ are operating points and frequency of the system, i.e., $\left(f, V_{d}, V_{q}, I_{d}, I_{q}\right)$ and $\boldsymbol{y}_{s}$ are the corresponding admittance, i.e., $\left(Y_{d d}, Y_{d q}, Y_{q d}, Y_{q q}\right)$. Similarly, the fieldmeasured impedance data in the target domain is denoted as $D_{t}$ $=\left\{\left(\boldsymbol{x}_{t 1}, \boldsymbol{y}_{t 1}\right), \ldots,\left(\boldsymbol{x}_{t m}, \boldsymbol{y}_{t m}\right)\right\}$, where $m<n$. In the transfer learning setting, if the data in the source and target domain follow the same distribution, i.e., $\boldsymbol{X}_{s} \neq \boldsymbol{X}_{t}, P\left(\boldsymbol{X}_{s}\right)=P\left(\boldsymbol{X}_{t}\right)$, the transferred admittance model can achieve a satisfactory application accuracy [15]. The distribution of the measured data and the simulated data are guaranteed the same in this paper.

\section{B. Framework description}

The framework of the proposed transfer learning based impedance identification method is shown in Fig. 4. The implementation of the method are divided as the offline phase and online phase.

In the offline phase, first, the simulation model is established in Matlab/Simulink, the simulated impedance data are obtained with the impedance measurement technique in the simulation. Second, the structure model that is derived in 
IEEE TechRxiv

Section II is used to restructure the ANN structure, which can reduce the demand for simulated data. After that, the offline ANN-based multi-operating-point impedance model is trained with the initialized ANN structure and the fed simulated impedance data.

In the online phase, the field impedance data of the new converter VSC I with the impedance measurement technique are fed into the offline-trained impedance model, where the parameters and the structure remain from the offline phase. As mentioned in Section III. B, the distribution of the fielded data should be the same. After the training, the multi-operatingpoint impedance model of the VSC I are achieved.

\section{IMPLEMENTATION OF TRANSFER LEARNING BASED IMPEDANCE IDENTIFICATION}

To implement the transfer learning based impedance identification method, the $d q$-impedance measurement is proposed to generate the dataset used for ANN training. And the ANN structure is generated with the analytical structure model derived in Section II.

\section{A. Dq-impedance measurement}

The $d q$-impedance measurement is proposed in variable operating points and frequency to obtain the dataset considering the variation of the operating points in different frequency.

Fig. 5 shows the diagram of $d q$-impedance measurement of the VSC. The current source-type perturbation is injected into the VSC at the point of common coupling (PCC). By extracting the response at PCC, the output impedance data of VSI can be calculated.

The detailed flowchart of the impedance measurement procedure in the $d q$-frame is shown in Fig. 6 that consists of perturbation injection, data processing, and impedance calculation.

The first step is to inject the perturbation at the specific frequency $f_{p}$ into the VSC system at PCC. Before the measurement, the magnitude of this perturbation signal has to be appropriately designed to extract the converter dynamics [16]. On the one hand, the magnitude of the perturbation has to be small to ensure the steady state of the operating point of the VSC system is stable. On the other hand, it has to be large to reject the influence of measurement noise. Generally, the magnitude of the perturbation is should be between $5 \%$ and $10 \%$ of steady-state values [17], [18].

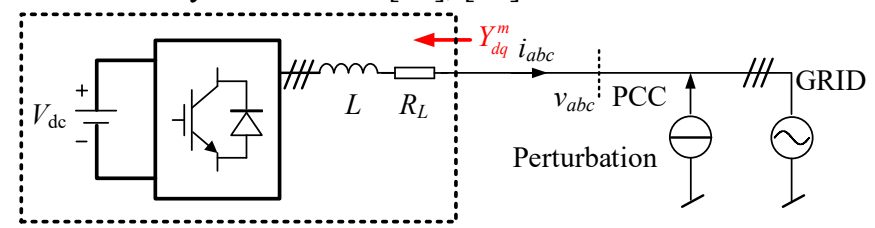

Fig. 5: $D q$-impedance measurement diagram.

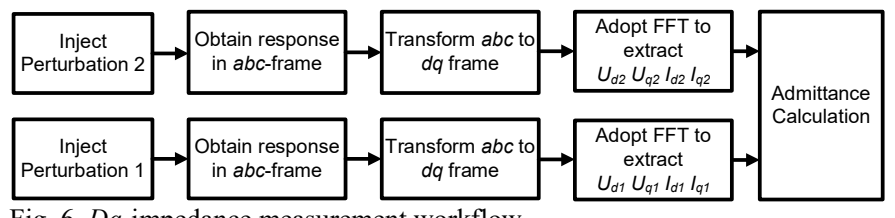

Fig. 6. $D q$-impedance measurement workflow.
The next step is to obtain the voltage and current response of the VSC at PCC and process the data. The measured output current and voltage of the VSC are the $a b c$-frame signals, the Park's transformation are used to transform the signal to $d q$ frame, i.e. $\boldsymbol{U}_{d 1}, \boldsymbol{U}_{q 1}, \boldsymbol{I}_{d 1}, \boldsymbol{I}_{q 1}$. The $\boldsymbol{U}_{d 1}, \boldsymbol{U}_{q 1}, \boldsymbol{I}_{d 1}, \boldsymbol{I}_{q 1}$ are the time series signal with the fixed sampling frequency. By applying the fast Fourier transform (FFT) algorithm, the voltage and current at the injected frequency can be extracted, i.e., $U_{d 1}\left(f_{p}\right)$, $U_{q 1}\left(f_{p}\right), I_{d 1}\left(f_{p}\right), D_{q 1}\left(f_{p}\right)$. To acquire the four entries of the impedance matrix $Y_{d q}^{m}\left(f_{p}\right)$, two linearly independent perturbations are injected [19]. Based on another extracted voltage and current at the injected frequency, i.e., $U_{d 2}\left(f_{p}\right)$, $U_{q 2}\left(f_{p}\right), I_{d 2}\left(f_{p}\right), D_{q 2}\left(f_{p}\right)$, the admittance of the VSC at specific frequency $f_{p}$ is calculated as follow

$$
\begin{aligned}
Y_{d q}^{m}\left(f_{p}\right) & =\left[\begin{array}{ll}
Y_{d d}\left(f_{p}\right) & Y_{d q}\left(f_{p}\right) \\
Y_{q d}\left(f_{p}\right) & Y_{q q}\left(f_{p}\right)
\end{array}\right] \\
& =\left[\begin{array}{ll}
U_{d 1}\left(f_{p}\right) & U_{d 2}\left(f_{p}\right) \\
U_{q 1}\left(f_{p}\right) & U_{q 2}\left(f_{p}\right)
\end{array}\right]^{-1}\left[\begin{array}{ll}
I_{d 1}\left(f_{p}\right) & I_{d 2}\left(f_{p}\right) \\
I_{q 1}\left(f_{p}\right) & I_{q 1}\left(f_{p}\right)
\end{array}\right]
\end{aligned}
$$

After the procedure, the magnitude and phase of the VSC impedance with a specific frequency $f_{p}$ and operating point are obtained. Repeating this procedure with by changing the frequency of the perturbation and operating point of VSC, the admittance dataset with variation are established, in which operating points and frequency are inputs while the corresponding magnitude and phase of the admittance are the outputs.

\section{B. ANN restructuration}

The conventional deep learning method needs a large amount of data to configure the ANN structure and the weights of the connection. If some parts of the ANN can be pre-decided, the demand of data can be greatly reduced. Based on the theoretical structure admittance model of VSC derived in Section II, this section shows the how to apply this structure model in the neural network restructuration.

The structure model in (15) can be represented as following:

$$
Y_{d q}^{m}(s)=\left[A_{d q}^{m}(s)-B_{d q}^{m}(s) \cdot V_{d q}^{m}-C_{d q}^{m}(s) \cdot I_{d q}^{m}\right] \cdot\left[D_{d q}^{m}(s)\right]^{-1}
$$

where:

$$
\begin{aligned}
& A_{d q}^{m}(s)=\left[\begin{array}{cc}
\frac{s}{L \cdot\left(s^{2}+\omega_{1}^{2}\right)} & \frac{\omega_{1}}{L \cdot\left(s^{2}+\omega_{1}^{2}\right)} \\
-\frac{\omega_{1}}{L \cdot\left(s^{2}+\omega_{1}^{2}\right)} & \frac{s}{L \cdot\left(s^{2}+\omega_{1}^{2}\right)}
\end{array}\right] \\
& B_{d q}^{m}(s)=\frac{s \cdot k_{p l l-p}+k_{p l l-i}}{s^{2}+\left(s \cdot k_{p l l-p}+k_{p l l-i}\right) v_{1 d}} \cdot k_{p}+\frac{k_{i}}{s} \cdot\left[\begin{array}{cc}
s & \omega_{1} \\
-\omega_{1} & s
\end{array}\right] \\
& C_{d q}^{m}(s)=\frac{1}{L\left(s^{2}+\omega_{1}^{2}\right)} \cdot \frac{s \cdot k_{p l l-p}+k_{p l l-i}}{s^{2}+\left(s \cdot k_{p l l-p}+k_{p l l-i}\right) v_{1 d}} \cdot e^{-1.5 T_{s} \cdot s} \cdot\left(k_{p}+\frac{k_{i}}{s}\right)\left[\begin{array}{cc}
s & \omega_{1} \\
-\omega_{1} & s
\end{array}\right] \\
& D_{d q}^{m}(s)=\left[\begin{array}{ll}
1+\frac{1}{L\left(s^{2}+\omega_{1}^{2}\right)} \cdot e^{-1.5 T_{s} \cdot s} \cdot\left(k_{p}+\frac{k_{i}}{s}\right) \cdot s & \frac{1}{L\left(s^{2}+\omega_{1}^{2}\right)} \cdot e^{-1.5 T_{s} \cdot s} \cdot\left(k_{p}+\frac{k_{i}}{s}\right) \cdot \omega_{1} \\
-\frac{1}{L\left(s^{2}+\omega_{1}^{2}\right)} \cdot e^{-1.5 T_{s} \cdot s} \cdot\left(k_{p}+\frac{k_{i}}{s}\right) \cdot \omega_{1} & 1+\frac{1}{L\left(s^{2}+\omega_{1}^{2}\right)} \cdot e^{-1.5 T_{s} \cdot s} \cdot\left(k_{p}+\frac{k_{i}}{s}\right) \cdot s
\end{array}\right] \\
& V_{s, d q}^{m}=\left[\begin{array}{ll}
0 & V_{s, d} \\
0 & V_{s, q}
\end{array}\right], I_{s, d q}^{m}=\left[\begin{array}{ll}
0 & I_{s, d} \\
0 & I_{s, q}
\end{array}\right]
\end{aligned}
$$




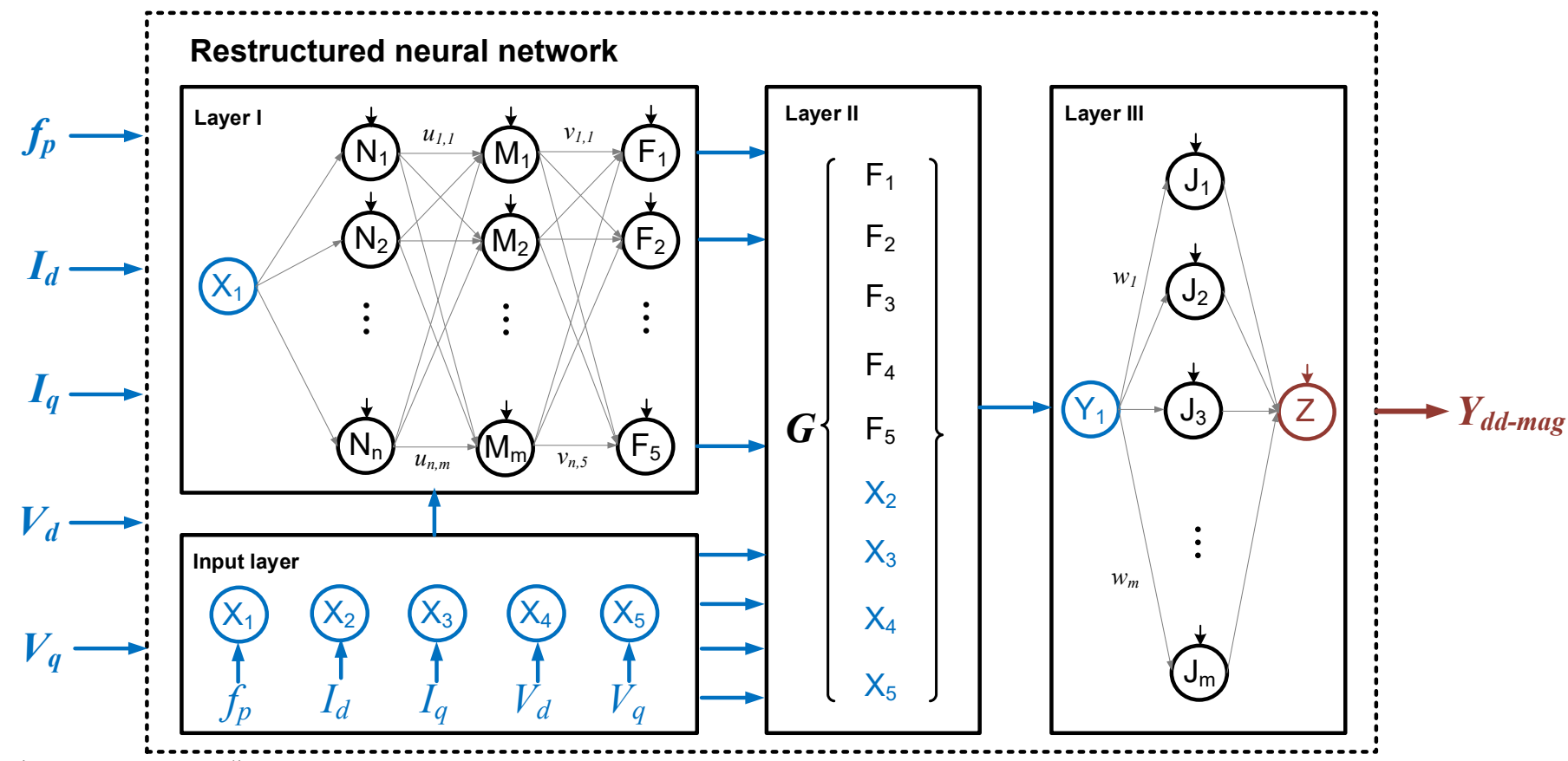

Fig. 7. ANN structure diagram.

As (17) shows, this structure model is naturally separated into the operating-point-dependent function and the frequency-dependent function. For simplification, (18) can be rewritten as:

$$
\begin{aligned}
& A_{d q}^{m}(s)=\left[\begin{array}{ll}
A_{d d}(s) & A_{d q}(s) \\
A_{q d}(s) & A_{q q}(s)
\end{array}\right], B_{d q}^{m}(s)=\left[\begin{array}{ll}
B_{d d}(s) & B_{d q}(s) \\
B_{q d}(s) & B_{q q}(s)
\end{array}\right], \\
& C_{d q}^{m}(s)=\left[\begin{array}{ll}
C_{d d}(s) & C_{d q}(s) \\
C_{q d}(s) & C_{q q}(s)
\end{array}\right],\left[D_{d q}^{m}(s)\right]^{-1}=\left[\begin{array}{ll}
D_{d d}^{-1}(s) & D_{d q}^{-1}(s) \\
D_{q d}^{-1}(s) & D_{q q}^{-1}(s)
\end{array}\right]
\end{aligned}
$$

Thus, (17) can be written as follows:

$$
\begin{aligned}
& Y_{d d}(s)=A_{d d}(s) D_{d d}^{-1}(s)+D_{q d}^{-1}(s) \cdot \\
& \quad\left(A_{q d}(s)-\left(V_{q} \cdot B_{d q}(s)+V_{d} \cdot B_{d d}(s)\right)-\left(I_{q} \cdot C_{d q}(s)+I_{d} \cdot C_{d d}(s)\right)\right) \\
& Y_{q d}(s)=A_{q d}(s) D_{d d}^{-1}(s)+D_{q d}^{-1}(s) \cdot \\
& \quad\left(A_{q q}(s)-\left(V_{d} \cdot B_{q d}(s)+V_{q} \cdot B_{q q}(s)\right)-\left(I_{d} \cdot C_{q d}(s)+I_{q} \cdot C_{q q}(s)\right)\right) \\
& Y_{d q}(s)=A_{d d}(s) D_{d q}^{-1}(s)+D_{q q}^{-1}(s) \cdot \\
& \quad\left(A_{q d}(s)-\left(V_{q} \cdot B_{d q}(s)+V_{d} \cdot B_{d d}(s)\right)-\left(I_{q} \cdot C_{d q}(s)+I_{d} \cdot C_{d d}(s)\right)\right) \\
& Y_{q q}(s)=A_{q d}(s) D_{d q}^{-1}(s)+D_{q q}^{-1}(s) \cdot \\
& \quad\left(A_{q q}(s)-\left(V_{d} \cdot B_{q d}(s)+V_{q} \cdot B_{q q}(s)\right)-\left(I_{d} \cdot C_{q d}(s)+I_{q} \cdot C_{q q}(s)\right)\right)
\end{aligned}
$$

Take $Y_{d d}$ as example:

$$
\begin{aligned}
Y_{d d}(s) & =F_{1}(s)-V_{q} \cdot F_{2}(s)-V_{d} \cdot F_{3}(s)-I_{q} \cdot F_{4}(s)-I_{d} \cdot F_{5}(s) \\
& =G\left[F_{1}(s), F_{2}(s), F_{3}(s), F_{4}(s), F_{5}(s), V_{q}, V_{d}, I_{q}, I_{d}\right]
\end{aligned}
$$

where $F_{1}(s), F_{2}(s), F_{3}(s), F_{4}(s), F_{5}(s)$ are the nonlinear functions of frequency while the linear calculation function $G$, that consists of these nonlinear functions and the operating points.

As the output of measured datasets consist of the magnitude and phase of the VSC admittance, the outputs of the neural network are written as follows:

$$
\begin{aligned}
& Y_{d d-\text { mag }}=\operatorname{Mag}\left[G\left[F_{1}(s), F_{2}(s), F_{3}(s), F_{4}(s), F_{5}(s), V_{q}, V_{d}, I_{q}, I_{d}\right]\right] \\
& Y_{d d-p h a}=\operatorname{Ang}\left[G\left[F_{1}(s), F_{2}(s), F_{3}(s), F_{4}(s), F_{5}(s), V_{q}, V_{d}, I_{q}, I_{d}\right]\right]
\end{aligned}
$$

where the nonlinear functions Mag and Ang can calculate the magnitude and phase of the impedance.

As a conclusion, the structure model of the impedance is separated into two nonlinear functions and one linear calculation function. Thus, the restructured ANN structure is shown in Fig. 6. This neural network is split into 3 layers. Layer $I$ is the nonlinear calculation layer, which generates the nonlinear output the nonlinear functions $F_{1}(s), F_{2}(s), F_{3}(s)$, $F_{4}(s), F_{5}(s)$. Layer II is the linear calculation layer, which represents the linear function $G$. Layer III is the magnitude and phase calculation layer, which generates the output of the whole ANN.

\section{CASE STUdy AND VALIDATION}

To verify the proposed transfer learning based impedance identification method, a case study of modeling the VSC systems using the physicals and measured data is designed.

A. Case study description

A three-phase VSC system marked as VSC II with the PI current controller and the PLL shown in Fig. 1 is the target VSC. A source VSC is designed with the same control structure and different parameters and is marked as VSC I. The detailed parameters of the VSCs are shown in Table I. 
IEEE TechRxiv

TABLE I. VSC PAERMETERS

\begin{tabular}{cccc}
\hline Symbol & Description & VSC I Value & VSC II Value \\
\hline$V_{d c}$ & Inverter input dc voltage & $700 \mathrm{~V}$ & $700 \mathrm{~V}$ \\
$f_{s w}$ & Switching frequency & $20 \mathrm{kHz}$ & $10 \mathrm{kHz}$ \\
$f_{0}$ & Line frequency & $50 \mathrm{~Hz}$ & $50 \mathrm{~Hz}$ \\
$L_{f}$ & Inductance of inverter output inductor & $1 \mathrm{mH}$ & $3 \mathrm{mH}$ \\
$R_{L f}$ & Parasitic resistance of inverter inductor & $0.3 \mathrm{~m} \Omega$ & $0.9 \mathrm{~m} \Omega$ \\
$K_{p_{-} i}$ & Proportional gain of current controller & 10.5 & 31.4 \\
$K_{i_{-} i}$ & Integral gain of current controller & 5900 & 16449 \\
$K_{p_{-} p l l}$ & Proportional gain of inverter PLL & 1.2 & 1.4 \\
$K_{i p l l}$ & Integral gain of inverter PLL & 257 & 324 \\
\hline
\end{tabular}

As mentioned in the framework descripted in Section III, the task is solving the impedance model of target VSC using the existing physical knowledge derived in Section IV and the model generated impedance model of the source VSC. The
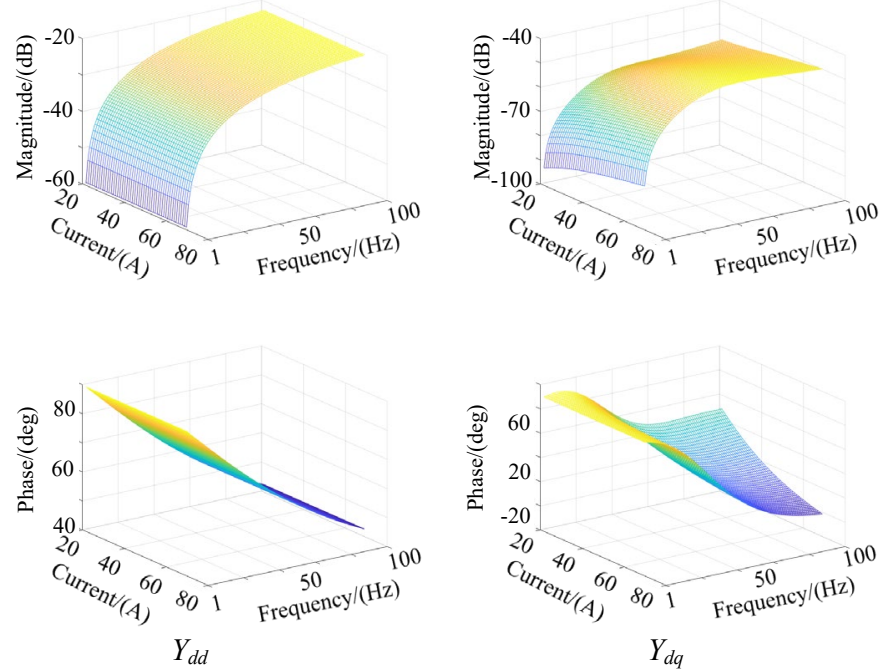

Fig. 8. Output admittance dataset of source VSC used for training.
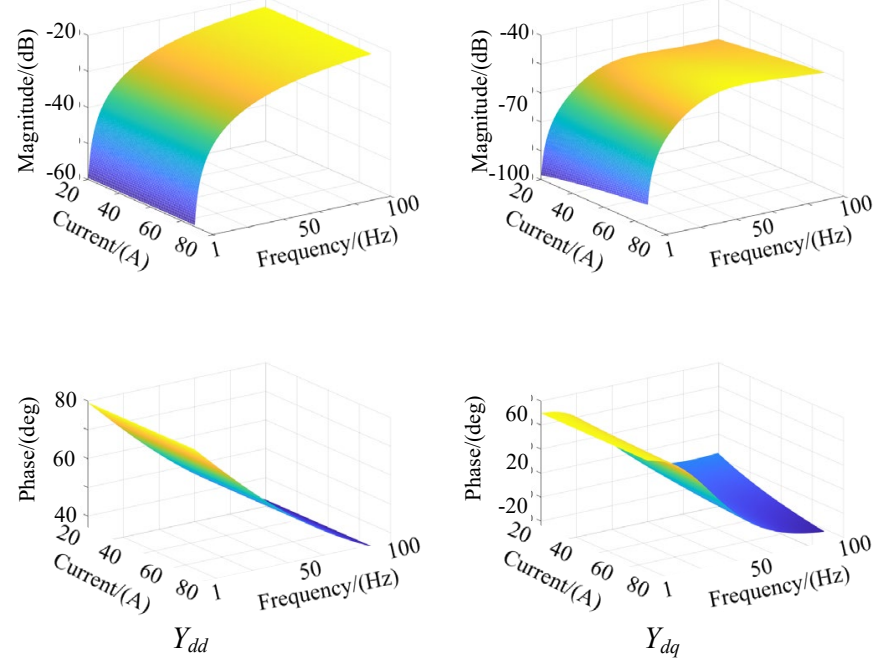

Fig. 10. Generated MOP-admittance model of source VSC. impedance data of the source VSC need is measured, then the impedance data are fed into the ANN structure and the impedance model of the source VSC is generated. To show the effectiveness of the proposed method, the comparison between
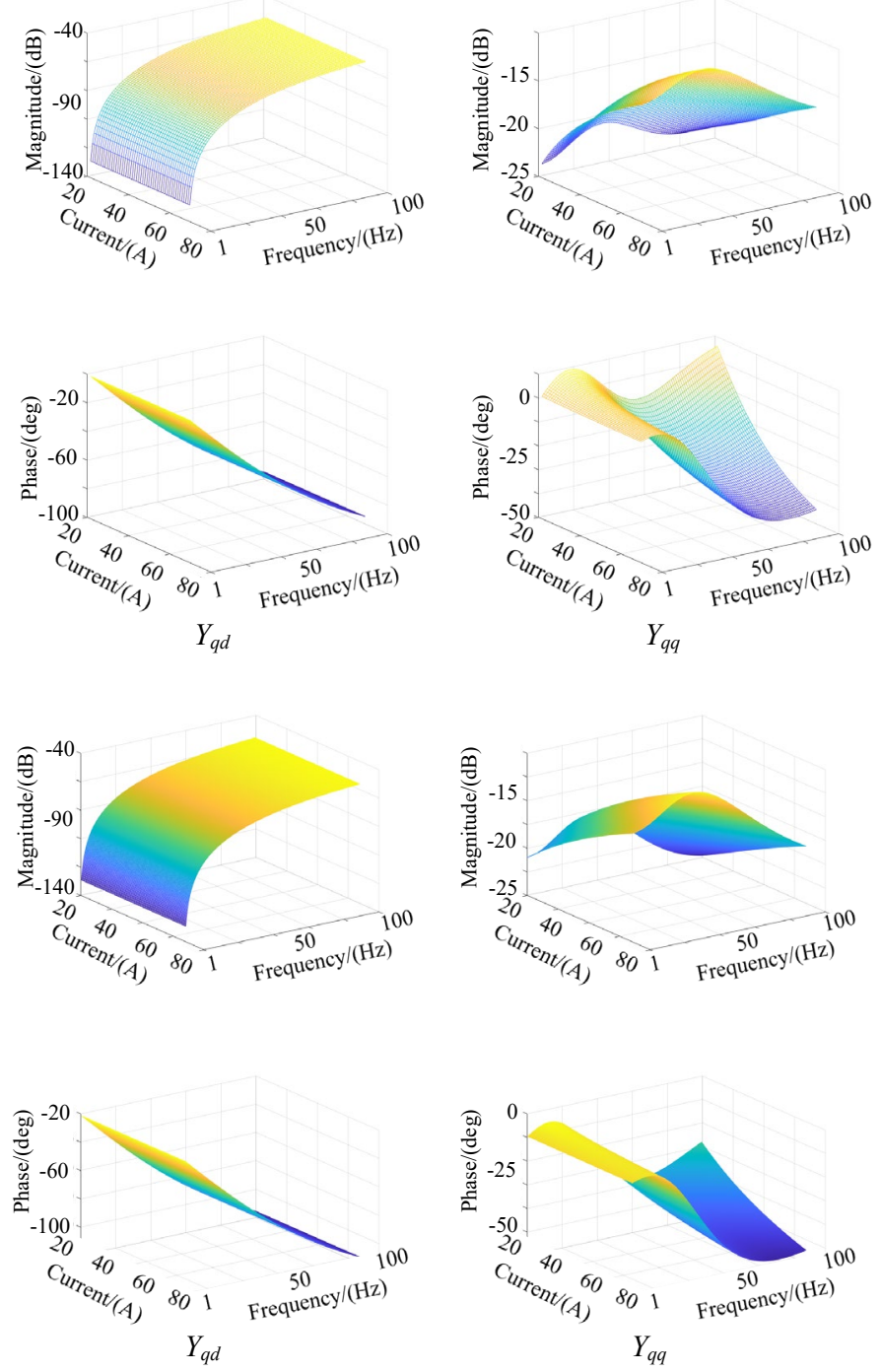


\section{IEEE TechRxiv}

the conventional ANN based identification method and the proposed transfer learning based identification method using the same measured impedance dataset is conducted.

\section{B. Transfer learning based impedance identification}

Before training the impedance model of the target VSC, the impedance model of source VSC need to be generated. The impedance measurement are conducted to obtain the training dataset. In this paper, the data are extracted by the simulation model, which is validated by the field measurements [20]. To make the result easily visible, only the operating point $I_{d}$ is variable. In the dataset, the frequency $f_{p}$ was swept from $1 \mathrm{~Hz}$ to $100 \mathrm{~Hz}$ with an interval of $1 \mathrm{~Hz}$, whereas the operating current $I_{d}$ was swept from $20 \mathrm{~A}$ to $70 \mathrm{~A}$ with an interval of $1 \mathrm{~A}$. The results are separated into $Y_{d d}, Y_{d q}, Y_{q d}, Y_{q q}$. The obtained output admittance dataset for training is shown in Fig. 9. Then the obtained dataset is fed into the restructured ANN. After the training process, the admittance model of the source VSC $Y_{V S C-I}$ is generated as shown in Fig. 10.

The model of source VSC is used to train the admittance model of the target VSC. In the impedance dataset of target VSC, the frequency $f_{p}$ was swept from $1 \mathrm{~Hz}$ to $101 \mathrm{~Hz}$ with an interval of $5 \mathrm{~Hz}$, whereas the operating current $I_{d}$ was swept
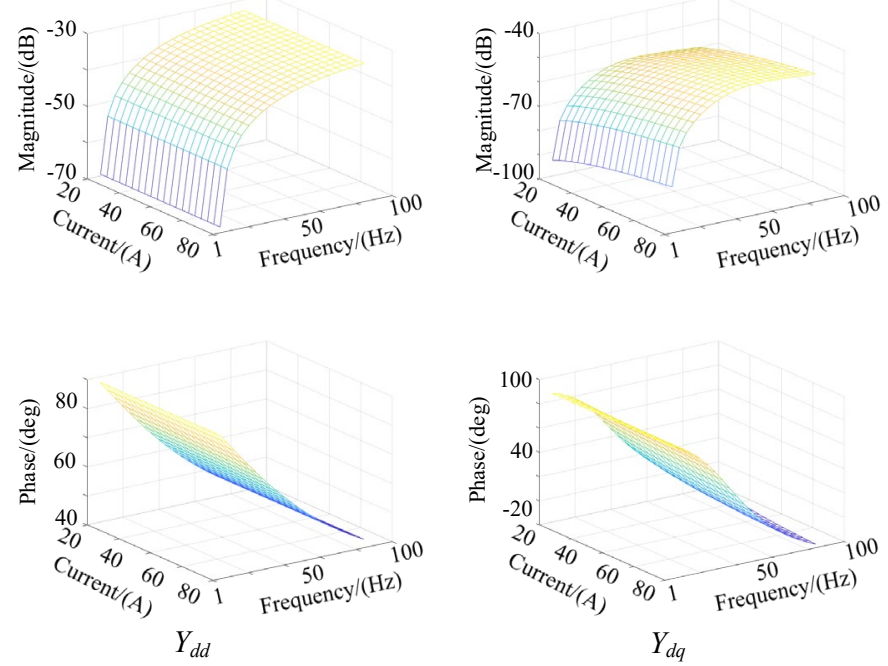

Fig. 11. Output admittance dataset of target VSC used for training.
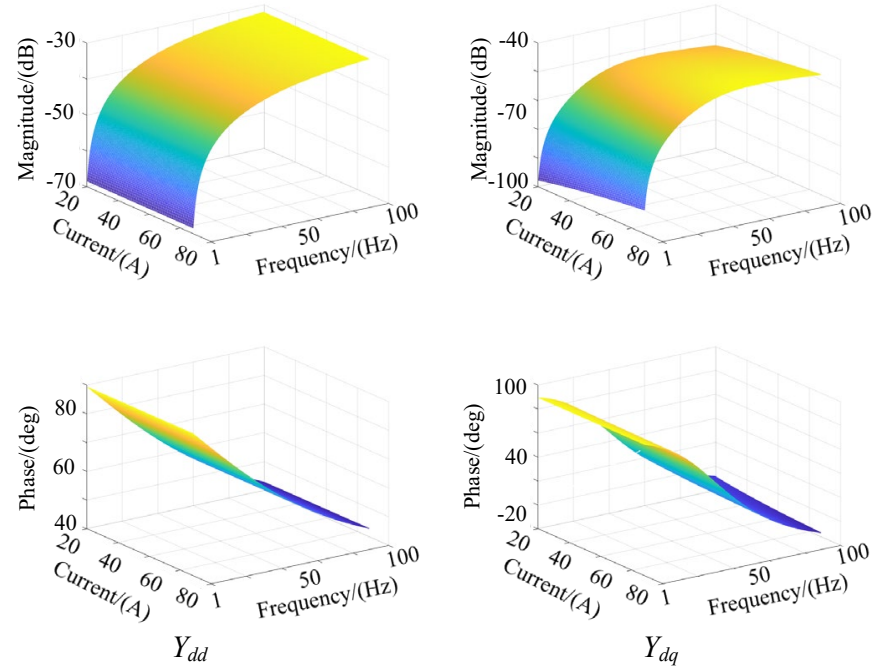

Fig. 12. Generated MOP-admittance model of target VSC. from $20 \mathrm{~A}$ to $70 \mathrm{~A}$ with an interval of $5 \mathrm{~A}$. In such case, only 200 data points are needed, which is shown in Fig. 11. Then feed the dataset into the admittance model $Y_{V S C-I}$. the admittance model of the target VSC $Y_{V S C-I I}$ is generated as shown in Fig. 12.

To verify the accuracy of the target VSC admittance model $Y_{V S C-I I}$, the generated ANN-based model needs to be compared with the verification dataset that is not used in training with the corresponding frequency $f_{p_{-} v}$ and operating current $I_{d_{-} v}$. In the verification dataset, the frequency $f_{p_{-}}$was swept from $1 \mathrm{~Hz}$ to $100 \mathrm{~Hz}$ with an interval of $0.1 \mathrm{~Hz}$, whereas the operating current $I_{d-v}$ was swept from 20 A to $70 \mathrm{~A}$ with an interval of 0.1 A. The corresponding errors between the verification data and the generated model are shown in Fig. 13, where the errors become large when the data used in training is sparse, yet the largest error is only $1 \%$ of the verification data. Hence, the proposed transfer learning based impedance identification method can accurately model the VSC considering the operating point variation.

\section{Comparison with the conventional ANN method}

The comparison between the conventional ANN method and the proposed transfer learning based identification method
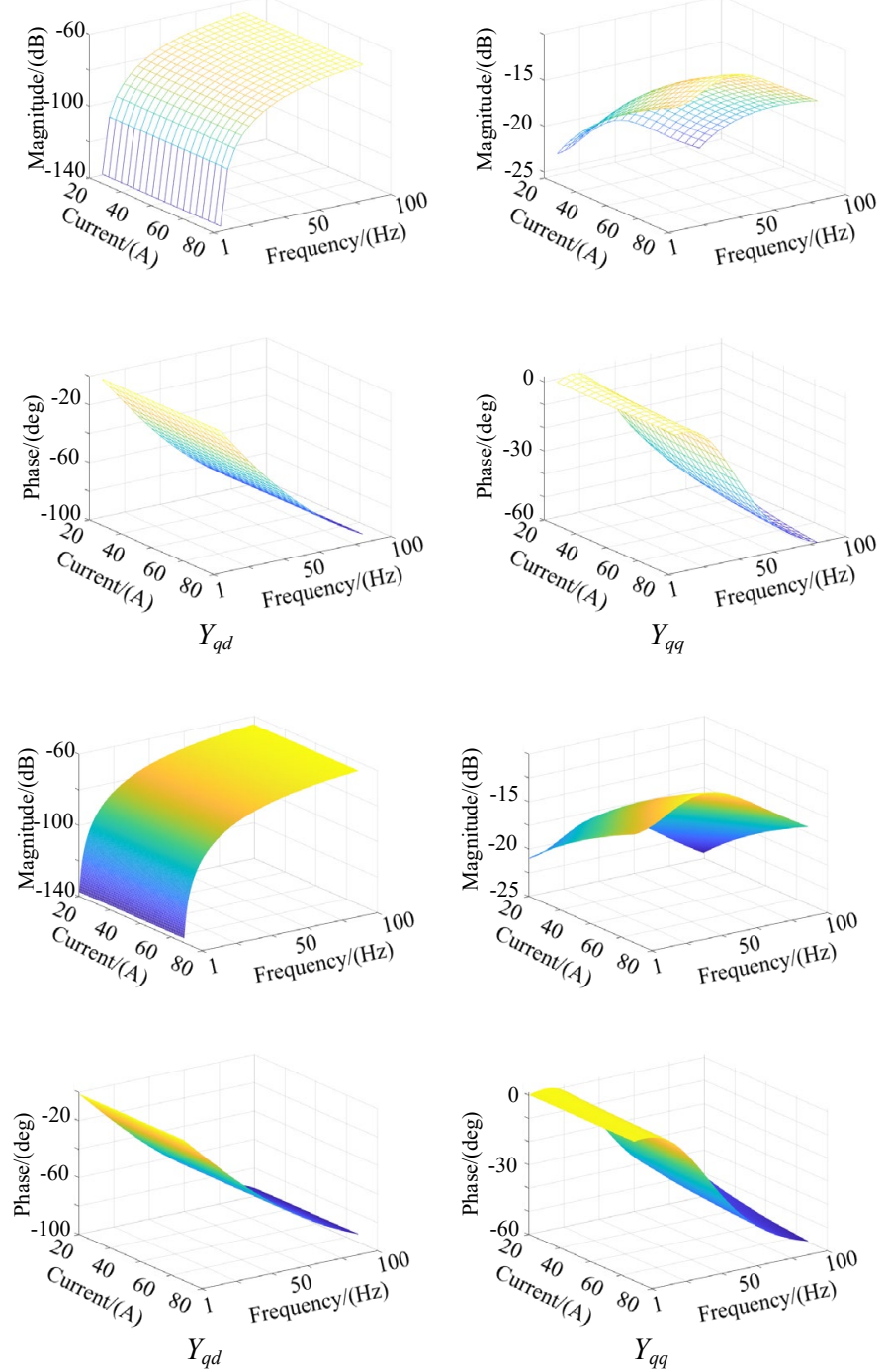

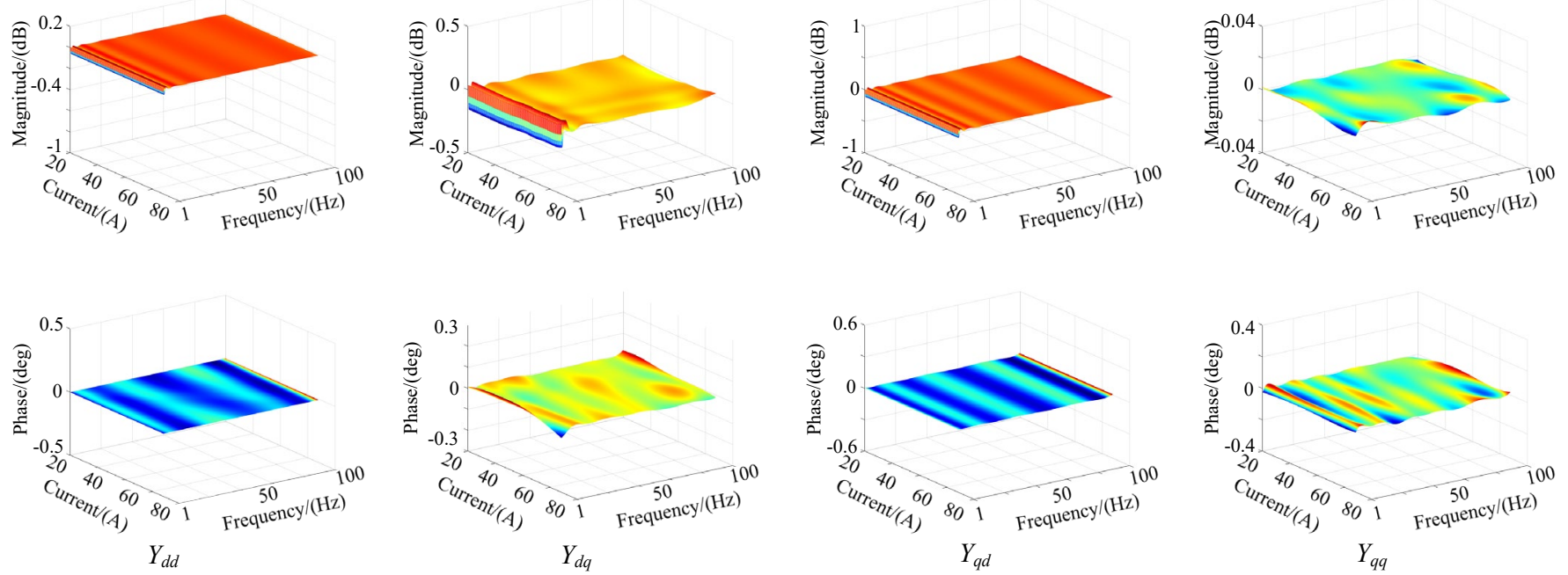

Fig. 13. Corresponding errors between multi-operating-point admittance model and verification data of proposed transfer learning method.
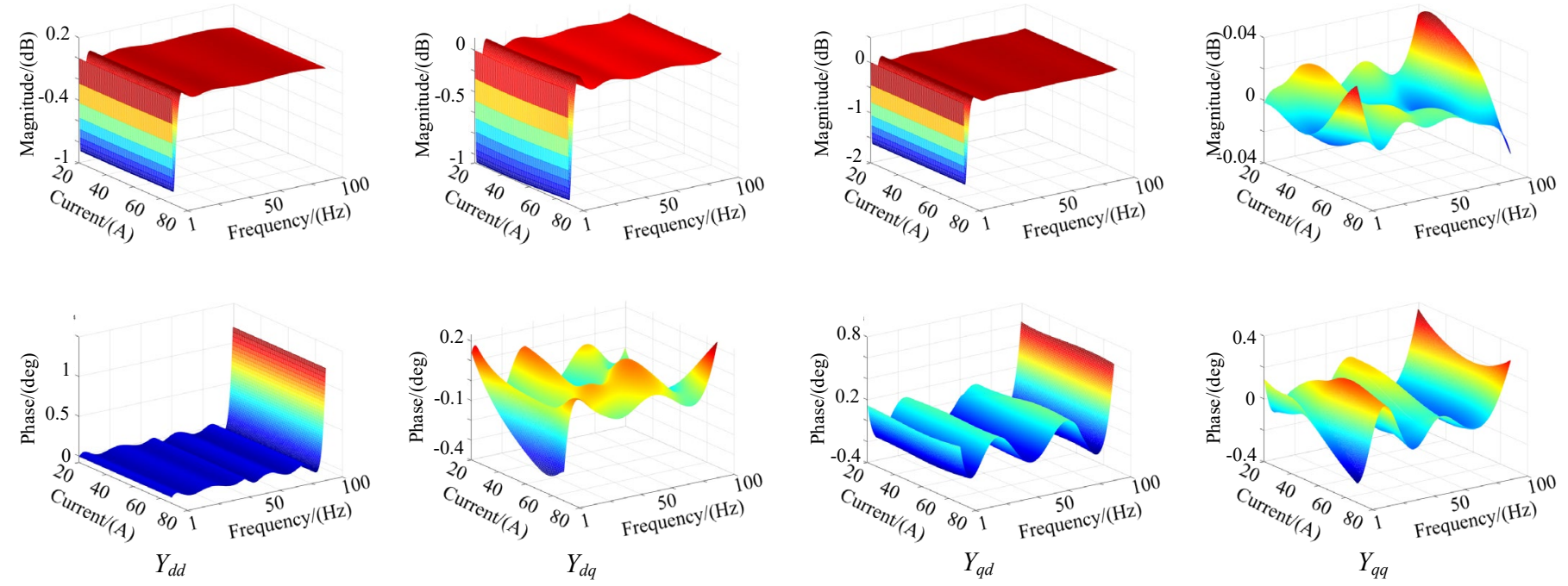

Fig. 14. Corresponding errors between multi-operating-point admittance model and verification data of conventional ANN method.

using the same measured impedance dataset is conducted to show the effectiveness of the transfer learning based method. The same dataset as shown in Fig. 11 are fed into a twohidden-layer random initialized ANN. After the training, the model $Y_{V S C-I I-O}$ is generated. To directly show the comparison result of two models. The corresponding errors between the verification data with the same distribution and the generated model are shown in Fig. 14.

Compared to the errors shown in Fig. 13, the conventional ANN method cannot have the good performance with the sparse dataset, where the proposed transfer learning based method can reduce the data amount requirement. Thus the proposed approach can address the disadvantages of the existing impedance identification methods in capturing the operating point-dependent feature of the impedance model.

\section{CONCLUSIONS}

This paper proposes a transfer learning based impedance identification method to address the data limitation issue in

impedance identification under changing operating conditions. An artificial neural network model is used to characterize the operating-point dependent impedance model. Considering the data amount limitation in this application, the transfer learning technique is employed in this paper. By combining the deep learning theory and impedance modeling knowledge, a transfer learning based impedance identification method is proposed. The proposed impedance identification method could be effectively applied for practical impedance identification and stability analysis with limited data. The comparison results can verify the superior of the proposed impedance identification method to existing methods.

\section{REFERENCES}

[1] F. Blaabjerg, R. Teodorescu, M. Liserre, and A. Timbus, "Overview of control and grid synchronization for distributed power generation systems," IEEE Trans. Ind. Electron., vol. 53, no. 5, pp. 1398-1409, Oct. 2006.

[2] X. Wang and F. Blaabjerg, "Harmonic Stability in Power ElectronicBased Power Systems: Concept, Modeling, and Analysis," IEEE Trans. Smart Grid, vol. 10, no. 3, pp. 2858-2870, May 2019. 


\section{IEEE TechRxiv}

[3] L. Harnefors, X. Wang, A. G. Yepes, and F. Blaabjerg, "Passivity based stability assessment of grid-connected VSCs-An overview," IEEE J. Emerging Sel. Topics Power Electron., vol. 4, no. 1, pp. 116-125, Mar. 2016.

[4] J. Sun, "Impedance-Based Stability Criterion for Grid-Connected Inverters," IEEE Trans. Power Electron., vol. 26, no. 11, pp. 3075-3078, Nov. 2011.

[5] D. Yang, X. Ruan and H. Wu, "Impedance Shaping of the GridConnected Inverter with LCL Filter to Improve Its Adaptability to the Weak Grid Condition," IEEE Trans. Power Electron., vol. 29, no. 11, pp. 5795-5805, Nov. 2014.

[6] X. Wang, F. Blaabjerg, and W. Wu, "Modeling and analysis of harmonic stability in an ac power-electronics-based power system," IEEE Trans. Power Electron., vol. 29, no. 12, pp. 6421-6432, Dec. 2014.

[7] M. Cespedes and J. Sun, "Three-phase impedance measurement for system stability analysis," 2013 IEEE 14th Workshop on Control and Modeling for Power Electronics (COMPEL), Salt Lake City, UT, 2013, pp. 1-6.

[8] X. Wang, L. Harnefors and F. Blaabjerg, "Unified Impedance Model of Grid-Connected Voltage-Source Converters," IEEE Trans. Power Electron., vol. 33, no. 2, pp. 1775-1787, Feb. 2018.

[9] A. Francés, R. Asensi, and J. Uceda, "Blackbox Polytopic Model With Dynamic Weighting Functions for DC-DC Converters," IEEE Access, vol. 7, pp. 160263-160273, Nov. 2019.

[10] M. Zhang, X. Wang, D. Yang and M. G. Christensen, “Artificial Neural Network based Identification of Multi-Operating-Point Impedance Model," IEEE Trans. Power Electron., vol. 36, no. 2, pp. 1231-1235, Feb. 2021.

[11] R.Y. Wang, D.M. Strong, and L.M. Guarascio, "Beyond Accuracy: What Data Quality Means to Data Consumers," (No. TDQM-94-10). Cambridge, Mass.: Total Data Quality Management Research Program, MIT Sloan School of Management, 1994.

[12] S. J. Pan and Q. Yang, "A Survey on Transfer Learning," IEEE Trans. Knowl. Data Eng., vol. 22, no. 10, pp. 1345-1359, Oct. 2010.

[13] D. Pan, X. Ruan, C. Bao, W. Li, and X. Wang, "Optimized controller design for LCL-type grid-connected inverter to achieve high robustness against grid-impedance variation," IEEE Trans. Ind. Electron., vol. 62, no. 3, pp. 1537-1547, Mar. 2015.

[14] L. Harnefors, M. Bongiorno, and S. Lundberg, "Input-admittance calculation and shaping for controlled voltage-source converters, " IEEE Trans. Ind. Electron., vol. 54, no. 6, pp. 3323-3334, Dec. 2007.

[15] S. J. Pan, I. W. Tsang, J. T. Kwok, and Q. Yang, "Domain adaptation via transfer component analysis," IEEE Trans. Neural Netw., vol. 22, no. 2, pp. 199-210, Feb. 2011.

[16] B. Miao, R. Zane and D. Maksimovic, "System identification of power converters with digital control through cross-correlation methods," IEEE Trans. Power Electron., vol. 20, no. 5, pp. 1093-1099, Sep. 2005.

[17] A. Riccobono, M. Mirz, and A. Monti, "Noninvasive online parametric identification of three-phase AC power impedances to assess the stability of grid-tied power electronic inverters in LV networks," IEEE J. Emerg.Sel. Top. Power Electron., vol. 6, no. 2, pp. 629-647, June. 2018.

[18] Z. Shen, M.Jaksic, B.Zhou, P.Mattavelli, J.Verhulst, M.Belkhayat, "Analysis of Phase Locked Loop (PLL) influence on DQ impedance measurement in three-phase AC systems," in Proc. Appl. Power Electron. Conf. Expo., Mar. 2013, pp. 939-945.

[19] G. Francis, R. Burgos, D. Boroyevich, F. Wang and K. Karimi, "An algorithm and implementation system for measuring impedance in the D-Q domain," 2011 IEEE Energy Conversion Congress and Exposition, Phoenix, AZ, 2011, pp. 3221-3228.

[20] H. Gong, D. Yang and X. Wang, "Impact of Nonlinear Dynamics on Converter DQ Impedance Measurement," in Proc. Control and Modeling for Power Electronics (COMPEL), June 2019, pp. 1-6. 